\title{
Acidosis during Reoxygenation Has an Early Detrimental Effect on Neuronal Metabolic Activity
}

\author{
ELISABETH FRØYLAND, FLEMMING WIBRAND, RUNAR ALMAAS, INGVILD DALEN, \\ JULIE K. LINDSTAD, AND TERJE ROOTWELT
}

Department of Pediatric Research [E.F., R.A., J.K.L., T.R.], Rikshospitalet University Clinic, N-0027 Oslo, Norway, The John F. Kennedy Institute [F.W.], DK-2600 Glostrup, Denmark, Department of Pediatrics

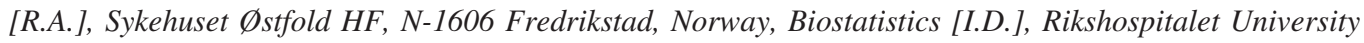
Clinic, N-0027 Oslo, Norway

\begin{abstract}
We recently showed that acidosis is protective during hypoxia and detrimental during reoxygenation. We hypothesized that the detrimental effect of acidosis during reoxygenation was due to a negative effect on mitochondrial function. Human postmitotic NT2-N neurons were exposed to $3 \mathrm{~h}$ of hypoxia and glucose deprivation and then reoxygenated for $0,1,4,9$, or $21 \mathrm{~h}$. The detrimental effect of acidotic reoxygenation on metabolic activity was evident already after $1 \mathrm{~h}$ of reoxygenation, when MTT [3-(4, 5-dimethylthiazol-2-yl)-2, 5-diphenyltetrazolium bromide] reduction (percentage of normoxic controls) was significantly higher in cells reoxygenated with neutral compared with acidotic medium both after acidotic hypoxia $(83 \pm 26 \%$ versus $67 \pm$ $27 \%, p=0.006)$ and after neutral hypoxia $(51 \pm 12 \%$ versus 41 $\pm 7 \%, p=0.005$ ). Hypoxanthine, a marker of cellular energy failure, increased more with acidotic compared with neutral reoxygenation both after acidotic hypoxia (after $21 \mathrm{~h}: 7.7 \pm 2.7$ versus $3.1 \pm 1.9 \mu \mathrm{M}, p<0.001)$ and after neutral hypoxia $(10.4$ \pm 2.6 versus $7.9 \pm 2.8 \mu \mathrm{M}, p=0.001)$. During hypoxia and reoxygenation, there was an earlier reduction in the activity of
\end{abstract}

\section{ABSTRACT}

complex IV compared with complexes II + III, and the ratio between these complexes fell during the first hour of reoxygenation. The reduction in complex IV activity was alleviated with acidotic hypoxia. Acidosis during reoxygenation, however, had no effect on the activity of either complex IV or complexes II + III. We conclude that acidosis during hypoxia increases neuronal survival and preserves complex IV activity. Acidosis during reoxygenation has an early detrimental effect on metabolic activity, but this is not mediated through an effect on the mitochondrial complexes IV or II+III. (Pediatr Res 57: 488493, 2005)
COX, cytochrome $c$ oxidase
Abbreviations
DMEM, Dulbecco's modified Eagle medium
LDH, lactate dehydrogenase
MTT, 3-(4, 5-dimethylthiazol-2-yl)-2, 5-diphenyltetrazolium bromide NMDA, $N$-methyl-D-aspartate

We recently showed that acidosis is protective during hypoxia and detrimental during reoxygenation in a human NT2-N neuronal culture (1). Several mechanisms with opposing effects may be involved. In our hypoxia model, cell death is mediated through NMDA and non-NMDA receptors, and pretreatment with MK-801 offers almost complete protection (1-4). Whereas the protective effect of acidosis during hypoxia is probably to a large extent mediated through an inhibitory effect

Received January 23, 2004; accepted August 18, 2004.

Correspondence: Elisabeth Frøyland, Department of Pediatric Research, Rikshospitalet University Clinic, N-0027 Oslo, Norway; e-mail: elisabeth.frøyland@medisin.uio.no

E.F. is a fellow at the University of Oslo (Norway). This project was also supported by the Norwegian Research Council, the Norwegian Society for Cardiovascular Diseases, The Laerdal Foundation for Acute Medicine, The Norwegian Air Ambulance, Medinnova, Anders Jahres Foundation, and the Blix Family Foundation.

DOI: 10.1203/01.PDR.0000155946.82230.2E on glutaminergic NMDA receptors $(1,5)$, the mechanisms behind the detrimental effect of acidosis during reoxygenation are less clear. Acidosis causes a $50 \%$ increase in free radical formation in the current model (1). However, as we have previously found a rather limited role for free radicals in hypoxic cell death of NT2-N neurons, this may not sufficiently explain the deleterious effect of acidosis during reoxygenation (3).

Abnormalities in mitochondrial function and mitochondrial (mt) DNA have been reported in several neurodegenerative diseases $(6,7)$, and mitochondria are important for both apoptotic and necrotic cell death (8). In perinatal hypoxic-ischemic insults, a delayed secondary energy failure may occur after an initial normalization of the energy status of the brain (9). A transient recovery and secondary decline in mitochondrial function have been demonstrated in many animal studies 
$(10,11)$. This may suggest that conditions during reperfusion can have important effects on mitochondrial function. We hypothesized that the detrimental effect of acidosis during reoxygenation was mediated through a deleterious effect on mitochondrial function.

Abe et al. (12) showed an early onset and a progressive reduction of the partially mitochondrially encoded COX-I mRNA levels and COX activity (complex IV) in contrast to a more stable nuclearly encoded succinate dehydrogenase activity (complex II) after an ischemic insult in gerbil neurons. We therefore also wanted to study the effects of hypoxia and acidosis on these complexes. Our model with hypoxia and glucose deprivation in human NT2-N "neurons" enabled us to separate the effects of acidosis during hypoxia and reoxygenation.

\section{MATERIALS AND METHODS}

Materials. MTT, bovine heart cytochrome $c$, and n-dodecyl- $\beta$-D-maltoside were purchased from Sigma Chemical (St. Louis, MO).

Hypoxia-reoxygenation. NT2 cells were prepared as previously described $(2,4)$. The hypoxia and glucose deprivation experiments were started 3 or $4 \mathrm{~d}$ after the last change of medium. Each well was examined microscopically at least $30 \mathrm{~min}$ before use. DMEM without glucose, with $100 \mathrm{IU} / \mathrm{mL}$ penicillin and $100 \mu \mathrm{g} / \mathrm{mL}$ streptomycin, was bubbled for a minimum of 5 min with $5 \%$ $\mathrm{CO}_{2}$ and $95 \% \mathrm{~N}_{2}$ and heated to $37^{\circ} \mathrm{C}$. In the experiments with acidosis, $\mathrm{HCl}$ was added to adjust $\mathrm{pH}$ to 5.9. Immediately before the experiment, the wells were washed twice with $0.5 \mathrm{~mL}$ PBS, and $0.5 \mathrm{~mL}$ of deoxygenated medium was added to each well. The cells were then placed in a preheated, humid anaerobic chamber (GasPak 150, Becton Dickinson, Sparks, MD). Inside the chamber hydrogen was generated with a Gaspak Plus envelope containing palladium catalyst to remove trace amounts of oxygen (BD Biosciences, Franklin Lakes, NJ). Vacuum was applied to the chamber for $25 \mathrm{~s}(20-25$ inches of $\mathrm{Hg}$ ) and the chamber was filled with $5 \% \mathrm{CO}_{2}$ and $95 \% \mathrm{~N}_{2}$. This procedure was performed four times. We have previously shown that this procedure reduces oxygen concentrations to $0.1-0.2 \%$ after the fourth gas exchange, falling to $<0.1 \%$ within $30-60 \mathrm{~min}$, and that these low oxygen levels are maintained thereafter (2). The chamber was then placed in an incubator at $37^{\circ} \mathrm{C}$ for $3 \mathrm{~h}$. Some experiments were stopped immediately after $3 \mathrm{~h}$ of hypoxia. In the remaining experiments, the medium was changed to a new medium containing glucose to a final concentration of $5.5 \mathrm{mM}$ with a $\mathrm{pH}$ of 5.9 or 7.4. The cells were then returned to the normoxic incubator for 1, 4, 9 , or $21 \mathrm{~h}$ before the experiments were ended. Thus, we had four experimental groups with neutral or acidotic medium during hypoxia and/or reoxygenation: $\mathrm{AN}=$ acidotic medium during hypoxia and neutral medium during reoxygenation, $\mathrm{AA}=$ acidotic medium during both hypoxia and reoxygenation, $\mathrm{NN}=$ neutral medium during hypoxia and reoxygenation, and NA = neutral medium during hypoxia and acidotic medium during reoxygenation. The four control groups (also labeled AN, AA, NN, and NA) received exactly the same treatment as the hypoxic groups, except that the DMEM added was with glucose and had been bubbled for $5 \mathrm{~min}$ with room air and that the chamber was flushed with room air after each vacuum. These experiments were terminated at $3+0 \mathrm{~h}$ or $3+21 \mathrm{~h}$ of sham treatment.

MTT assay. To monitor the metabolic activity, we used an MTT assay (13). MTT, $0.5 \mathrm{mg} / \mathrm{mL}$, was dissolved in PBS containing $5.5 \mathrm{mM}$ glucose. The medium was aspirated, the wells were washed twice with $0.5 \mathrm{~mL}$ PBS, and 0.5 $\mathrm{mL}$ of the MTT solution was added. The plates were then returned to the normoxic incubator. After $60 \mathrm{~min}$, the MTT solution was aspirated, and the formed crystals were dissolved with $0.5 \mathrm{~mL}$ DMSO. Absorbance measurements were done with a Titertek Multiskan Plus MK 11 MTT assay (Labsystem, Helsinki, Finland) connected to a computer with the program GENESIS (Thermo Labsystems, Egelsbach, Germany). Absorbance was read at 570 $\mathrm{nm}$ and background at $660 \mathrm{~nm}$ was subtracted. MTT reduction was calculated as percentage of MTT reduction in normoxic control cells with neutral medium at $0 \mathrm{~h}$.

Hypoxanthine. Accumulation of the ATP breakdown product hypoxanthine is an indicator of cellular energy failure (14), and it has been shown to react abruptly to changes in oxygenation status (15). Hypoxanthine was therefore used as a marker of cellular energy status. Supernatants were collected at 0,1 , 4,9 , and $21 \mathrm{~h}$ after the end of hypoxia and frozen at $-20^{\circ} \mathrm{C}$. Hypoxanthine was analyzed with HPLC on a reversed-phase C18 column (Pecosphere-5C C18,
$0.46 \times 15.0 \mathrm{~cm}$, PerkinElmer Instruments, Norwalk, CT). Next, $0.01 \mathrm{M}$ $\mathrm{KH}_{2} \mathrm{PO}_{4}(\mathrm{pH} 4.2)$ was used as a mobile phase with a flow rate of $0.8 \mathrm{~mL} / \mathrm{min}$. The eluting compounds were detected at $254 \mathrm{~nm}$ (4).

$\boldsymbol{L D H}$. LDH release measured by a cytotoxicity detection kit from Roche (Basel, Switzerland) was used as a marker of cell lysis. Supernatants were collected at $0,1,4,9$, and $21 \mathrm{~h}$ after the end of hypoxia and $0.5 \mathrm{~mL} \mathrm{H}_{2} \mathrm{O}$ was added to each well to lyse the cells. Measurements of LDH were done with a Titertek Multiskan Plus MK 11 ELISA reader connected to a computer with the program GENESIS. LDH release in percentage of total LDH was then calculated as supernatant/(supernatant + lysate). We found that acidosis markedly affected the stability of LDH in the supernatant (data not shown). LDH was therefore only measured in the two groups that were neutral during reoxygenation (the $\mathrm{NN}$ and $\mathrm{AN}$ groups).

Measurements of mitochondrial complexes IV and II+III activities. Three wells were washed with PBS, scraped off, and collected together in a total volume of $0.5 \mathrm{~mL}$ PBS. The cells were centrifuged and the pellet frozen at $-70^{\circ} \mathrm{C}$. The cells were later resuspended in $0.4 \mathrm{~mL}$ of ice-cold STE buffer ( $250 \mathrm{mM}$ sucrose, $10 \mathrm{mM}$ Tris/HCl, $2 \mathrm{mM}$ EDTA, pH 7.4) and disrupted with a tight-fitting Teflon-glass homogenizer. The homogenate was then frozen in liquid nitrogen and stored at $-80^{\circ} \mathrm{C}$ until enzyme measurements could be made. Enzyme assays were performed at $30^{\circ} \mathrm{C}$ in a final volume of $0.2 \mathrm{~mL}$ using a SPECTRAmax PLUS ${ }^{384}$ Micro plate Spectrophotometer (Molecular Devices, Sunnyvale, CA). COX (complex IV) activity was determined by monitoring the initial rate of ferrocytochrome $c(200 \mu \mathrm{M})$ oxidation at the wavelength pair $510-535 \mathrm{~nm}$ (16) in a medium containing $50 \mathrm{mM}$ potassium phosphate $(\mathrm{pH} 7.0)$ and $0.02 \% \mathrm{n}$-dodecyl- $\beta$-D-maltoside. Succinatecytochrome $c$ reductase (complex II+III) was measured by following the reduction of ferricytochrome $c$ at $550 \mathrm{~nm}$ with $580 \mathrm{~nm}$ as the reference wavelength (17). Enzyme activities were calculated as nanomoles cytochrome $c$ oxidized (complex IV) or reduced (complex II + III) per minute per milligram of cell protein. We also calculated the ratio of complex IV/II+III to reduce analytic variance and to demonstrate the different effect of hypoxiareoxygenation on these complexes (18). The protein concentration was determined using a BCA kit (Pierce Chemical, Rockford, IL).

Statistics. All values are given as mean $\pm \mathrm{SD}$. Whenever one treatment was tested in more than one well in one plate, the average was used in the analyses. Wells within the same 12-well culture plate were considered matched, as they had been exposed to identical conditions during $9 \mathrm{wk}$ of culture and during the hypoxia experiments, except for the tested treatment, i.e. different $\mathrm{pH}$. We always tested all four different $\mathrm{pH}$ treatments within the same plate, and paired samples $t$ tests were therefore used to compare these. Comparisons between various time points for a given treatment group (i.e. between wells on independent cell plates) were performed by independent samples $t$ tests. Welch correction was applied in cases where variances were found to be significantly different by means of Levene's test for equality of variances. Except for LDH, statistical comparisons were only done at 0,1 , and $21 \mathrm{~h}$. At $0 \mathrm{~h}$, acidotic and neutral hypoxia were compared. At 1 and $21 \mathrm{~h}$, acidotic and neutral reoxygenation were compared. Finally, $1 \mathrm{~h}$ was compared with $0 \mathrm{~h}$ within each $\mathrm{pH}$ group. A two-tailed $p<0.05$ was considered statistically significant. All statistical analyses were performed with SPSS for Windows 11 (SPSS Inc., Chicago. IL).

\section{RESULTS}

Acidosis is protective during hypoxia. After $3 \mathrm{~h}$ of hypoxia and glucose deprivation, MTT reduction (percentage of normoxic controls) was significantly lower in the cells with neutral medium during hypoxia than in the cells that had been acidotic $(47 \pm 15 \%, n=10$ versus $68 \pm 20 \%, n=10 ; p<0.001)$ (Fig. 1). This protective effect of acidosis during hypoxia on MTT reduction was maintained during $21 \mathrm{~h}$ of reoxygenation. Release of the ATP breakdown product hypoxanthine to the supernatant was used as an indicator of cellular energy failure (14). Hypoxanthine was significantly lower in the group that had been acidotic during hypoxia $(5.2 \pm 2.3 \mu \mathrm{M}, n=12)$ compared with the group that had been kept neutral $(8.6 \pm 2.4$ $\mu \mathrm{M}, n=12, p<0.001$ ), reflecting a less compromised energy situation in the acidotic group (Fig. 2). After 9 and $21 \mathrm{~h}$ of reoxygenation, LDH release was significantly reduced with acidotic compared with neutral hypoxia (Fig. 3). Finally, at the end of hypoxia, complex IV activity was significantly higher 


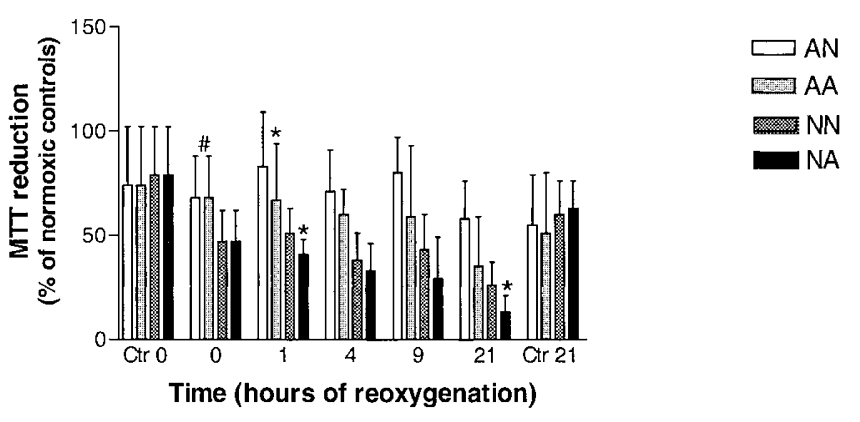

Figure 1. MTT reduction in percentage of normoxic neutral controls from 0 to $21 \mathrm{~h}$ of reoxygenation after $3 \mathrm{~h}$ of combined hypoxia and glucose deprivation and at 0 and $21 \mathrm{~h}$ in normoxic sham-treated controls (Ctr 0 and Ctr 21). Four different $\mathrm{pH}$ groups: $A N$, acidotic during hypoxia and neutral during reoxygenation; $A A$, acidotic during both hypoxia and reoxygenation; $N N$, neutral during both hypoxia and reoxygenation; and $N A$, neutral during hypoxia and acidotic during reoxygenation. At $0 \mathrm{~h}$, only two groups existed (acidotic or neutral medium), both for hypoxia and controls. Pair-wise identical bars are therefore displayed at this time. Results are given as mean $\pm \mathrm{SD}, n=$ 7-10. \#p $<0.001$ acidotic $v s$ neutral hypoxia at $0 \mathrm{~h} ; * p<0.05$ acidotic $v s$ neutral reoxygenation (AA $v s$ AN and NA $v s \mathrm{NN}$ ) at $1 \mathrm{~h}$ and (NN $v s \mathrm{NA}) 21 \mathrm{~h}$.

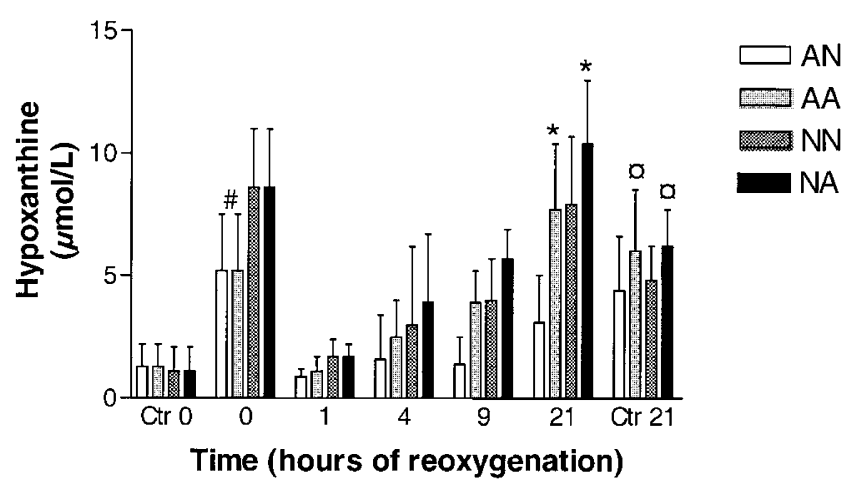

Figure 2. Hypoxanthine from 0 to $21 \mathrm{~h}$ of reoxygenation after $3 \mathrm{~h}$ of combined hypoxia and glucose deprivation and at 0 and $21 \mathrm{~h}$ in normoxic sham-treated controls (Ctr 0 and Ctr 21). Note that after the hypoxia, all wells were washed and all hypoxanthine was therefore removed before reoxygenation. Four different $\mathrm{pH}$ groups: $A N$, acidotic during hypoxia and neutral during reoxygenation; $A A$, acidotic during both hypoxia and reoxygenation; $N N$, neutral during both hypoxia and reoxygenation; and $N A$, neutral during hypoxia and acidotic during reoxygenation. At $0 \mathrm{~h}$, only two groups existed (acidotic or neutral medium), both for hypoxia and controls. Pair-wise identical bars are therefore displayed at this time. Results are given as mean $\pm \mathrm{SD}, n=$ 9-15. $\# p<0.001$ acidotic $v s$ neutral hypoxia; ${ }^{*} p<0.001$ acidotic $v s$ neutral reoxygenation (both $\mathrm{AA} v s \mathrm{AN}$ and $\mathrm{NA} v s \mathrm{NN}$ ) at $21 \mathrm{~h}$. $x p<0.05$ for normoxic sham-treated controls, acidotic $v s$ neutral reoxygenation (both AA $v s$ $\mathrm{AN}$ and NA $v s \mathrm{NN}$ ) at $21 \mathrm{~h}$.

after acidotic compared with neutral hypoxia $(174 \pm 26 \mathrm{nmol} /$ (min $\cdot \mathrm{mg}$ protein), $n=4$ versus $135 \pm 15 \mathrm{nmol} /(\mathrm{min} \cdot \mathrm{mg}$ protein), $n=4, p=0.02$ ) (Fig. 4). Complex II+III activity at this time was similar to controls and not significantly different between the neutral and acidotic groups (Fig. 5).

Acidosis is detrimental during reoxygenation. Already at $1 \mathrm{~h}$ of reoxygenation, MTT reduction was significantly higher in the AN versus the AA group $(83 \pm 26 \%, n=8$ versus 67 $\pm 27 \%, n=8 ; p=0.006)$ and in the NN group compared with the NA group $(51 \pm 12 \%, n=8$ versus $41 \pm 7 \%, n=8 ; p=$ $0.005)$. These differences were maintained during reoxygenation and after $21 \mathrm{~h}$ MTT reduction was higher in the AN group

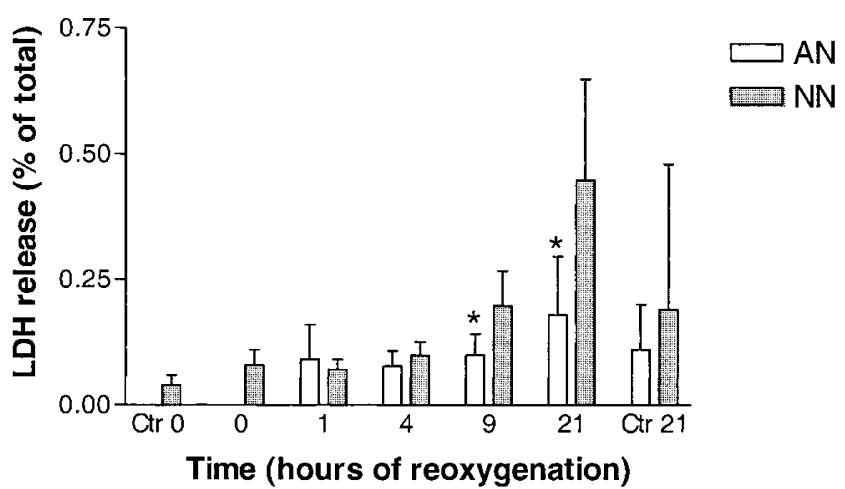

Figure 3. LDH release in percentage of total LDH from 0 to $21 \mathrm{~h}$ of reoxygenation after $3 \mathrm{~h}$ of combined hypoxia and glucose deprivation and at 0 and $21 \mathrm{~h}$ in normoxic sham-treated controls (Ctr 0 and Ctr 21). Because acidosis markedly affected the stability of LDH in the supernatant, LDH was only measured in the two groups that were neutral during reoxygenation and at $0 \mathrm{~h}$ only in the neutral group. Two different $\mathrm{pH}$ groups: $A N$, acidotic during hypoxia and neutral during reoxygenation; $N N$, neutral during both hypoxia and reoxygenation. Results are given as mean $\pm \mathrm{SD}, n=6-10 . * p<0.05 \mathrm{AN}$ vs NN.

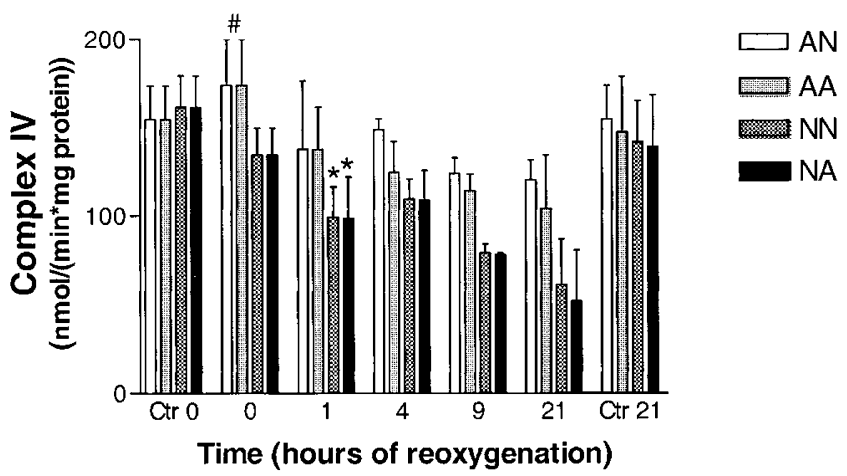

Figure 4. Complex IV activity from 0 to $21 \mathrm{~h}$ of reoxygenation after $3 \mathrm{~h}$ of combined hypoxia and glucose deprivation and at 0 and $21 \mathrm{~h}$ in normoxic sham-treated controls (Ctr 0 and $\mathrm{Ctr} 21$ ). Four different $\mathrm{pH}$ groups: $A N$, acidotic during hypoxia and neutral during reoxygenation; $A A$, acidotic during both hypoxia and reoxygenation; $N N$, neutral during both hypoxia and reoxygenation; and $N A$, neutral during hypoxia and acidotic during reoxygenation. At $0 \mathrm{~h}$, only two groups existed (acidotic or neutral medium), both for hypoxia and controls. Pair-wise identical bars are therefore displayed at this time. Results are given as mean $\pm \mathrm{SD}, n=3-5$. $\# p<0.05$ acidotic $v s$ neutral hypoxia at $0 \mathrm{~h} ; * p<0.05,1$ vs $0 \mathrm{~h}$ for $\mathrm{NN}$ and NA.

compared with the AA group $(58 \pm 18 \%, n=7$ versus $35 \pm$ $24 \%, n=7 ; p=0.056$ ) and in the NN group compared with the NA group $(26 \pm 11 \%, n=7$ versus $13 \pm 8 \%, n=7 ; p=$ 0.005) (Fig. 1).

After hypoxia, all wells received new medium so that all the hypoxanthine released during hypoxia was removed, and all groups started at "zero." During reoxygenation, hypoxanthine progressively accumulated in all four groups (Fig. 2), with significantly greater increase after $21 \mathrm{~h}$ with acidotic reoxygenation compared with neutral reoxygenation, both after neutral hypoxia $(10.4 \pm 2.6 \mu \mathrm{M}, n=11$ versus $7.9 \pm 2.8 \mu \mathrm{M}, n$ $=11 ; p=0.001)$ and after acidotic hypoxia $(7.7 \pm 2.7 \mu \mathrm{M}$, $n=11$ versus $3.1 \pm 1.9 \mu \mathrm{M}, n=11 ; p<0.001)$, reflecting a more compromised energy situation with acidotic reoxygenation. 


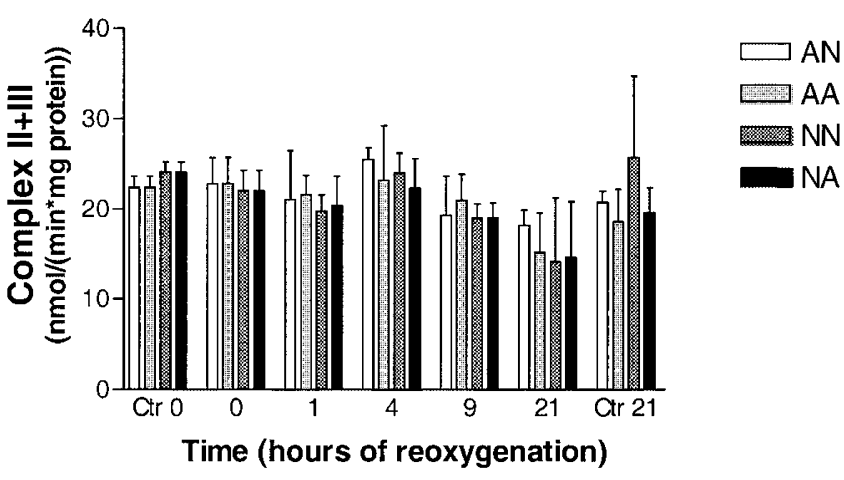

Figure 5. Complex II $+\mathrm{III}$ activity from 0 to $21 \mathrm{~h}$ of reoxygenation after $3 \mathrm{~h}$ of combined hypoxia and glucose deprivation and at 0 and $21 \mathrm{~h}$ in normoxic sham-treated controls (Ctr 0 and $\mathrm{Ctr} 21$ ). Four different $\mathrm{pH}$ groups: $A N$, acidotic during hypoxia and neutral during reoxygenation; $A A$, acidotic during both hypoxia and reoxygenation; $N N$, neutral during both hypoxia and reoxygenation; and $N A$, neutral during hypoxia and acidotic during reoxygenation. At $0 \mathrm{~h}$, only two groups existed (acidotic or neutral medium), both for hypoxia and controls. Pair-wise identical bars are therefore displayed at this time. Results are given as mean $\pm \mathrm{SD}, n=3-5$.

Different response to hypoxia and reoxygenation of complex IV and complex II+III. Complex IV activity was reduced already at the end of hypoxia in cells that were not protected by acidosis during hypoxia and fell significantly further during the first hour of reoxygenation in the two groups that had been neutral during hypoxia [the NN group: from 135 $\pm 15 \mathrm{nmol} /(\min \cdot \mathrm{mg}$ protein $), n=4$, to $99 \pm 17 \mathrm{nmol} /(\min$ - $\mathrm{mg}$ protein), $n=5, p=0.01$; the NA group: from $135 \pm 15$ $\mathrm{nmol} /(\min \cdot \mathrm{mg}$ protein), $n=4$, to $99 \pm 23 \mathrm{nmol} /(\mathrm{min} \cdot \mathrm{mg}$ protein), $n=5, p=0.03$ ] (Fig. 4). The activity of complex II + III, on the other hand, showed a slower decline (Fig. 5). To further demonstrate the different responses of complex IV and complex II+III, we calculated the ratio of complex IV to complex II+III (Fig. 6). This method has been shown to reduce the effect of random variation and to demonstrate more clearly a change of a single complex or activity (18). During the first hour of reoxygenation, there was a significant reduction in this

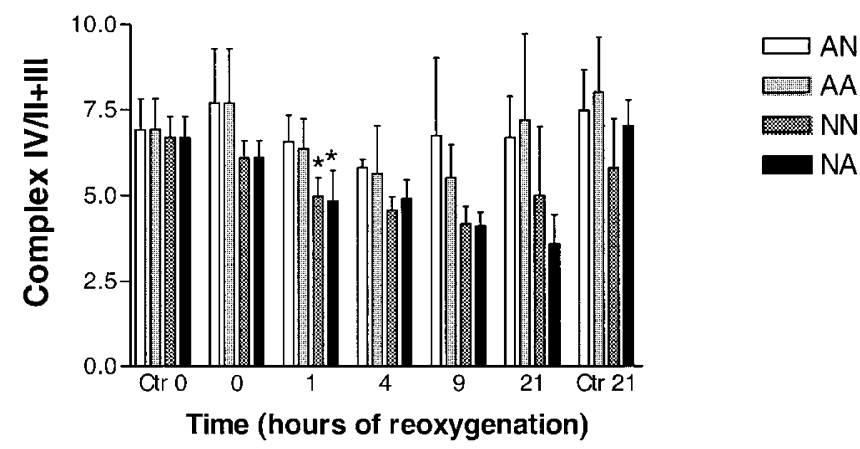

Figure 6. The ratio between complex IV and II + III activities from 0 to $21 \mathrm{~h}$ of reoxygenation after $3 \mathrm{~h}$ of combined hypoxia and glucose deprivation and at 0 and $21 \mathrm{~h}$ in normoxic sham-treated controls (Ctr 0 and Ctr 21). Four different $\mathrm{pH}$ groups: $A N$, acidotic during hypoxia and neutral during reoxygenation; $A A$, acidotic during both hypoxia and reoxygenation; $N N$, neutral during both hypoxia and reoxygenation; and $N A$, neutral during hypoxia and acidotic during reoxygenation. At $0 \mathrm{~h}$, only two groups existed (acidotic or neutral medium), both for hypoxia and controls. Pair-wise identical bars are therefore displayed at this time. Results are given as mean $\pm \mathrm{SD}, n=3-5$. * $p$ $<0.05,1$ vs $0 \mathrm{~h}$ for $\mathrm{NN}$ and NA. ratio in the two groups that had been neutral during hypoxia (the $\mathrm{NN}$ group: from $6.1 \pm 0.5, n=4$, to $5.0 \pm 0.5, n=5, p$ $=0.02$; the NA group: from $6.1 \pm 0.5, n=4$, to $4.8 \pm 0.9, n$ $=5, p=0.04$ ) (Fig. 6). A similar but not significant change was also seen in the two groups that had been protected by acidosis during hypoxia.

Effect of acidosis on the activities of complex IV and complex II+III during reoxygenation. Neither complex IV activity (Fig. 4), complex II+III activity (Fig. 5), nor the ratio between these (Fig. 6) were significantly affected by $\mathrm{pH}$ during reoxygenation.

Effects of acidotic reoxygenation in control cells. Acidosis during reoxygenation had no effect on MTT reduction (Fig. 1) or complex IV or II+III activities (Fig. 4 and 5) in control cells. Hypoxanthine values, however, were moderately but significantly increased by acidosis during reoxygenation (Fig. 2).

\section{DISCUSSION}

In the present study, we have confirmed the recent finding (1) that acidosis is protective during hypoxia and detrimental during reoxygenation. Acidotic reoxygenation was further found to have an early detrimental effect on MTT reduction and lead to a progressively increased hypoxanthine accumulation indicative of a more severe energy failure.

Under normoglycemic conditions, brain $\mathrm{pH}$ may fall below 6.2 during hypoxia-ischemia, and under hyperglycemic conditions, $\mathrm{pH}$ may fall below 5.6 (19). Several experiments have demonstrated aggravation of hypoxic-ischemic damage by hyperglycemia and this has been interpreted as due to aggravated acidosis $(9,20)$. On the other hand, acidosis has been shown to reduce hypoxic damage in cell cultures, probably through inhibition of NMDA- $(1,5,21)$ and kainate- (5) mediated damage. Acidosis may therefore have diverse and opposing effects on neuronal function and survival. To study this further, we have developed a model where we are able to separate the effects of acidosis during hypoxia and reoxygenation.

The protective effect of acidosis during hypoxia was confirmed in the present experiment both by analysis of MTT reduction, LDH release, and hypoxanthine accumulation. It was probably to a large degree mediated through inhibition of NMDA receptor-mediated damage $(1,5,21)$. NMDA receptor stimulation is central to hypoxic cell death in our model (1-4), and blockade of NMDA receptors confers no additive protection to that given by acidosis alone (1). In the present study, we further found that acidosis during hypoxia preserved complex IV activity.

The detrimental effect of acidosis during reoxygenation on MTT reduction was apparent already after $1 \mathrm{~h}$, both in the groups that had been acidotic and those that had been neutral during hypoxia. We considered the possibility that acidosis negatively influenced the MTT assay itself, but the cells were washed twice with buffer before the assay and the assay was performed in a buffered solution. Furthermore, MTT reduction was unaffected by acidosis in the normoxic control experiments. In our previous article, vitality staining after $21 \mathrm{~h}$ 
showed increased cell death with acidotic reoxygenation, confirming that acidosis indeed affects survival (1).

The MTT results were corroborated by the hypoxanthine analyses. Hypoxanthine is a breakdown product from ATP metabolism, and it has been shown that its accumulation is an indicator of cellular energy failure $(14,15)$. Measurement of ATP levels is a more direct marker of energy level, but hypoxanthine was chosen as it could be analyzed in the supernatants from cells used for other analyses and is more stable than ATP. Previously, hypoxanthine has been shown to be highly negatively correlated with ATP (22). During reoxygenation, hypoxanthine increased more in the groups that were acidotic during reoxygenation compared with the neutral groups. This negative effect of acidosis on the cellular energy situation was apparent even in the control cells, with moderately higher hypoxanthine levels after $21 \mathrm{~h}$ of acidosis.

A possible aggravating effect of acidosis during reoxygenation could be through an increase in free radical formation $(1,23)$, but we have not found oxygen radicals to be important mediators of cell death in our model (3). Another possible detrimental effect of acidosis during reoxygenation could be through a negative effect on mitochondrial function. The present findings of an early detrimental effect on MTT reduction and progressive accumulation of hypoxanthine with acidotic reoxygenation are compatible with impaired mitochondrial function due to acidosis. However, MTT reduction is not specific for mitochondrial function as some reduction may occur outside the mitochondria in living cells $(13,24)$. The detrimental effect of acidosis during reoxygenation in our study was not due to a negative effect on the mitochondrial complexes IV or II+III.

In our study, the activity of complex IV was affected earlier than complex II+III, and this was confirmed with the analysis of the ratio of complex IV to complex II+III (18). This is in accordance with the findings of Abe et al. (12), who found an early onset and a progressive reduction of the partly mitochondrially encoded COX-I mRNA levels and COX activity (complex IV) in contrast to a more stable nuclearly encoded succinate dehydrogenase activity (complex II). Other animal studies have also found an early depression of complex IV activity $(25,26)$. This early onset and progressive disturbance of complex IV activity may cause failure of energy production, and we have previously shown that a combination of energy failure and glutamate stimulation is toxic to NT2-N neurons (2). Davey et al. (27) found that complex I, III, and IV activities in brain mitochondria had to be decreased by approximately $25 \%$, $80 \%$, and $70 \%$, respectively, before major changes in oxygen consumption and ATP synthesis were observed. In the present study, the reduction in complex IV activity at $21 \mathrm{~h}$ of reoxygenation in cells that had not been protected by acidosis during hypoxia was almost two thirds. This is comparable to the level that impairs ATP production. However, the reduction at previous time points was more modest and not by itself enough to explain the observed changes in MTT and hypoxanthine. It is therefore unclear whether the decline in mitochondrial function after hypoxia-ischemia in this study and previous studies contributes to the final neuronal damage or if it is a secondary event in the development of injury (28). In the study by Abe $e t$ al. (12), the early affection of complex IV compared with complex II was interpreted as due to a disturbance of mitochondrial DNA expression. As we analyzed a combined complex II+III activity, and complex III has one subunit that is mitochondrially encoded, in this respect our data must be interpreted with caution, although it has been shown that in our assay complex II is the major point of control and not complex III (29).

The finding that acidosis is detrimental during reoxygenation is consistent with the notion that NMDA receptor-mediated damage is less important during reoxygenation. In keeping with this, we have previously demonstrated that treatment with the NMDA receptor blocker MK-801 at the end of hypoxia did not reduce cell death (2).

There are several limitations to the findings in the present study. This was an in vitro study performed on a cell line, albeit with many neuronal characteristics (1). Also, as mentioned, MTT reduction is not specific for mitochondrial function, because some reduction may occur outside the mitochondria in living cells $(13,24)$ and hypoxanthine is similarly only a surrogate marker for cellular ATP levels.

In conclusion, in the present study we have confirmed that acidosis is protective during hypoxia and detrimental during reoxygenation. Acidotic reoxygenation was found to have an early detrimental effect on metabolic activity, measured as MTT reduction, and to cause an increased accumulation of hypoxanthine. Complex IV activity was affected earlier by hypoxia and reoxygenation than complex II+III. Whereas acidosis during hypoxia preserved complex IV activity, $\mathrm{pH}$ during reoxygenation did not significantly affect the activities of complexes II+III and IV. Changes in complexes II+III and IV could therefore not explain the detrimental effects of acidosis during reoxygenation.

\section{REFERENCES}

1. Almaas R, Pytte M, Lindstad JK, Wright M, Saugstad OD, Pleasure D, Rootwelt T 2003 Acidosis has opposite effects on neuronal survival during hypoxia and reoxygenation. J Neurochem 84:1018-1027

2. Rootwelt T, Dunn M, Yudkoff M, Itoh T, Almaas R, Pleasure D 1998 Hypoxic cell death in human NT2-N neurons: involvement of NMDA and non- NMDA glutamate receptors. J Neurochem 71:1544-1553

3. Almaas R, Saugstad OD, Pleasure D, Rootwelt T 2002 Neuronal formation of free radicals plays a minor role in hypoxic cell death in human NT2-N neurons. Pediatr Res 51:136-143

4. Almaas R, Saugstad OD, Pleasure D, Rootwelt T 2000 Effect of barbiturates on hydroxyl radicals, lipid peroxidation, and hypoxic cell death in human NT2-N neurons. Anesthesiology 92:764-774

5. Giffard RG, Monyer H, Christine CW, Choi DW 1990 Acidosis reduces NMDA receptor activation, glutamate neurotoxicity, and oxygen-glucose deprivation neuronal injury in cortical cultures. Brain Res 506:339-342

6. Ikebe S, Tanaka M, Ohno K, Sato W, Hattori K, Kondo T, Mizuno Y, Ozawa T 1990 Increase of deleted mitochondrial DNA in the striatum in Parkinson's disease and senescence. Biochem Biophys Res Commun 170:1044-1048

7. Parker WD Jr, Boyson SJ, Luder AS, Parks JK 1990 Evidence for a defect in NADH: ubiquinone oxidoreductase (complex I) in Huntington's disease. Neurology 40:12311234

8. Kruman II, Mattson MP 1999 Pivotal role of mitochondrial calcium uptake in neural cell apoptosis and necrosis. J Neurochem 72:529-540

9. Lorek A, Takei Y, Cady EB, Wyatt JS, Penrice J, Edwards AD, Peebles D, Wylezinska M, Owen-Reece H, Kirkbride V 1994 Delayed ("secondary") cerebral energy failure after acute hypoxia-ischemia in the newborn piglet: continuous 48-hour studies by phosphorus magnetic resonance spectroscopy. Pediatr Res 36:699-706

10. Nakai A, Taniuchi Y, Asakura H, Yokota A, Koshino T, Araki T 2000 Secondary mitochondrial dysfunction after transient intrauterine ischemia in the fetal rat brain. J Obstet Gynaecol Res 26:383-387

11. Li PA, Kristian T, He QP, Siesjo BK 2000 Cyclosporin A enhances survival, ameliorates brain damage, and prevents secondary mitochondrial dysfunction after a 30-minute period of transient cerebral ischemia. Exp Neurol 165:153-163 
12. Abe K, Aoki M, Kawagoe J, Yoshida T, Hattori A, Kogure K, Itoyama Y 1995 Ischemic delayed neuronal death. A mitochondrial hypothesis. Stroke 26:1478-1489

13. Liu Y, Peterson DA, Kimura H, Schubert D 1997 Mechanism of cellular 3-(4,5 dimethylthiazol-2-yl)-2,5-diphenyltetrazolium bromide (MTT) reduction. J Neurochem 69:581-593

14. Saugstad OD 1988 Hypoxanthine as an indicator of hypoxia: its role in health and disease through free radical production. Pediatr Res 23:143-150

15. Almaas R, Sundar TB, Rootwelt T, Oyasaeter S, Saugstad OD 1997 Plasma hypoxanthine reacts more abruptly to changes in oxygenation than base deficit and uric acid in newborn piglets. J Perinat Med 25:353-360

16. Wiedemann FR, Vielhaber S, Schroder R, Elger CE, Kunz WS 2000 Evaluation of methods for the determination of mitochondrial respiratory chain enzyme activities in human skeletal muscle samples. Anal Biochem 279:55-60

17. Birch-Machin MA, Turnbull DM 2001 Assaying mitochondrial respiratory complex activity in mitochondria isolated from human cells and tissues. Methods Cell Bio 65:97-117

18. Munnich A, Rustin P 2001 Clinical spectrum and diagnosis of mitochondrial disorders. Am J Med Genet 106:4-17

19. Siemkowicz E, Hansen AJ 1981 Brain extracellular ion composition and EEG activity following 10 minutes ischemia in normo- and hyperglycemic rats. Stroke 12:236-240

20. Roth SC, Edwards AD, Cady EB, Delpy DT, Wyatt JS, Azzopardi D, Baudin J, Townsend J, Stewart AL, Reynolds EO 1992 Relation between cerebral oxidative metabolism following birth asphyxia, and neurodevelopmental outcome and brain growth at one year. Dev Med Child Neurol 34:285-295
21. Tombaugh GC, Sapolsky RM 1990 Mild acidosis protects hippocampal neurons from injury induced by oxygen and glucose deprivation. Brain Res 506:343-345

22. Simmonds RJ, Coade SB, Harkness RA, Drury I, Hytten FE 1982 Nucleotide, nucleoside and purine base concentrations in human placentae. Placentae 3:29-38

23. Siesjo BK, Bendek G, Koide T, Westerberg E, Wieloch T 1985 Influence of acidosis on lipid peroxidation in brain tissues in vitro. J Cereb Blood Flow Metab $5: 253-258$

24. Berridge MV, Tan AS 1993 Characterization of the cellular reduction of 3-(4,5dimethylthiazol-2-yl)-2,5-diphenyltetrazolium bromide (MTT): subcellular localization, substrate dependence, and involvement of mitochondrial electron transport in MTT reduction. Arch Biochem Biophys 303:474-482

25. Nelson C, Silverstein FS 1994 Acute disruption of cytochrome oxidase activity in brain in a perinatal rat stroke model. Pediatr Res 36:12-19

26. Dimlich RV, Showers MJ, Shipley MT 1990 Densitometric analysis of cytochrome oxidase in ischemic rat brain. Brain Res 516:181-191

27. Davey GP, Peuchen S, Clark JB 1998 Energy thresholds in brain mitochondria Potential involvement in neurodegeneration. J Biol Chem 273:12753-12757

28. Wagner KR, Kleinholz M, Myers RE 1990 Delayed decreases in specific brain mitochondrial electron transfer complex activities and cytochrome concentrations following anoxia/ischemia. J Neurol Sci 100:142-151

29. Taylor RW, Birch-Machin MA, Bartlett K, Turnbull DM 1993 Succinate-cytochrome c reductase: assessment of its value in the investigation of defects of the respiratory chain. Biochim Biophys Acta 1181:261-265 